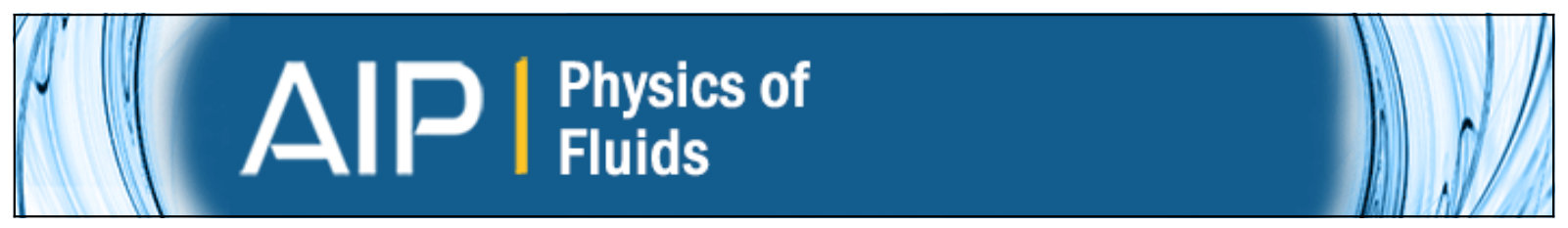

\title{
Analysis of quasi-static vapour bubble shape during growth and departure
}

Frédéric J. Lesage, James S. Cotton, and Anthony J. Robinson

Citation: Physics of Fluids (1994-present) 25, 067103 (2013); doi: 10.1063/1.4809795

View online: http://dx.doi.org/10.1063/1.4809795

View Table of Contents: http://scitation.aip.org/content/aip/journal/pof2/25/6?ver=pdfcov

Published by the AIP Publishing

Articles you may be interested in

Analysis of interfacial instability and multimode bubble formation in saturated boiling using coupled level set and volume-of-fluid approach

Phys. Fluids 26, 012105 (2014); 10.1063/1.4861760

Asymmetric interface temperature during vapor bubble growth

Appl. Phys. Lett. 103, 031602 (2013); 10.1063/1.4813561

Bubble motion and size variation during thermal migration with phase change

Phys. Fluids 25, 013302 (2013); 10.1063/1.4774329

Homogeneous bubble nucleation in liquids: The classical theory revisited

J. Chem. Phys. 118, 792 (2003); 10.1063/1.1525797

Dynamics of bubble growth on a heated surface under low gravity conditions

AIP Conf. Proc. 504, 751 (2000); 10.1063/1.1307229

\section{AlP Re-register for Table of Content Alerts}




\title{
Analysis of quasi-static vapour bubble shape during growth and departure
}

\author{
Frédéric J. Lesage, ${ }^{1,2, a)}$ James S. Cotton, ${ }^{2}$ and Anthony J. Robinson ${ }^{2,3}$ \\ ${ }^{1}$ Department of Mathematics, Cégep de l'Outaouais, Gatineau J8Y 6M4, Canada \\ ${ }^{2}$ Department of Mechanical Engineering, McMaster University, Hamilton L8S 4L7, Canada \\ ${ }^{3}$ Department of Mechanical Engineering, Trinity College, Dublin 2, Ireland
}

(Received 25 October 2012; accepted 26 April 2013; published online 14 June 2013)

\begin{abstract}
In an effort to better understand the physical mechanisms responsible for pool boiling heat transfer, a numerical solution to the capillary equation is used to describe bubble shape evolution. Indeed, any analysis of thermal transport due to nucleate pool boiling requires bubble frequency and volume predictions, which are intimately linked to bubble shape. To this end, a numerical treatment of the capillary equation is benchmarked to profiles measured from captured images of vapour bubble formations. The bubble growth is quasi-static in a quiescent liquid with a triple contact line fixed to the perimeter of a needle orifice. This investigation provides insight into the dependence the bubble shape evolution has on the physical mechanisms quantified in the Bond number with characteristic length equal to the cavity radius. (ㄷ $2013 \mathrm{AIP}$ Publishing LLC. [http://dx.doi.org/10.1063/1.4809795]
\end{abstract}

\section{INTRODUCTION}

Many industrial practices create large amounts of heat. Thermal control of the site, industrial instruments, and electrical components is vital to the industries running capacity and is managed with heat exchangers. For this reason, industrial needs have motivated a large number of studies of the underlying physics of nucleate pool boiling for their capacity to generate high heat transfer rates. These are intimately linked to the vapour bubbles, which form, grow, and depart at nucleation sites. In addition to evaporative cooling effects, fluid motions induced by bubble activity disrupt the thermal boundary layer in the vicinity of the bubbles causing enhanced mixing and improved heat transfer in these regions. ${ }^{1}$

Many empirical and mechanism-based correlations have been developed in order to predict nucleate pool boiling heat transfer rates. ${ }^{2-4}$ Since it is the bubble volume at detachment which dictates detachment frequency and influences the heat transfer rates, many detachment volume empirical correlations were developed by Refs. 5-8 and others. In these correlations, the varying parameter is a Bond number with the bubble spherical volume equivalent detachment radius as its characteristic length. In a comprehensive study comparing bubble detachment correlations with available data in the literature ${ }^{9}$ concluded that an empirical correlation developed by Ref. 10 had the best fit with a standard deviation of $45.4 \%$. An anomaly in these correlations is that the bubble is assumed to be spherical, implying an infinitesimally small point of contact with the heated element, while incorporating the Bond number, which implies that the surface tension plays a central role. Since the surface tension is a consequence of contact between the bubble and the heated element, the empirical correlations themselves suggest that the bubble shape is not spherical and has a significant contact with the bubble growth site, and that the nucleation cavity should be incorporated into bubble growth modeling.

In order to identify the Bond number as a parameter of the experimental conditions rather than the experimental results, in their bubble pinch-off study of adiabatic bubble growth, Ref. 11 defined the

a) Author to whom correspondence should be addressed. Electronic mail: Frederic.Lesage@cegepoutaouais.qc.ca 
Bond number with characteristic length equal to the orifice radius rather than the bubble detachment radius. Similarly, Ref. 12 used this definition of the Bond number to successfully develop a bubble volume detachment correlation for needle injected adiabatic bubble formations. In the present study, the characteristic length of the Bond number is set to be the radius of the cavity from which the bubble is issuing in order to include cavity size in the bubble shape analysis.

The present day difficulties lie in the fact that the empirical correlations fall off rapidly in accuracy once outside the range in which they were developed and the numerical simulations have a very high computational expense. In order to ease the calculations, numerical treatments of the problem often assume the bubble to be a sphere ${ }^{13,14}$ or a truncated spherical segment, ${ }^{15,16}$ or use other simplifying assumptions depending on the aim of the particular investigation. For example, in Refs. 17-23, the numerically simulated bubbles are modeled to be very large compared with the actual nucleation site to ensure that the vapour temperature remains at system pressure saturation temperature during the growth cycle. However, ebullition frequency and detachment volume are observed to vary from one nucleation site to another on a uniformly heated surface (e.g., see Ref. 2) implying that the characteristics of a nucleation site - namely, its radius - are central to the thermal transport capacity of bubble growth from that site. These above mentioned shape assumptions while alleviating the computational expense - compromise the thermal transport predictions.

In an effort to better understand bubble shape behaviour, Ref. 24 validated the shape evolving numerical solutions to the interface pressure balance represented in the capillary equation to gas diffusion bubbles. In these experiments, bubble formation took place at an artificial nucleation cavity of radius $1.04 \mathrm{~mm}$ in saturated carbonated water. The numerical solution to the capillary equation was further benchmarked by Ref. 25 by comparing the resulting profiles with processed images of quasi-static air injection bubbles with an orifice of radius $0.259 \mathrm{~mm}$.

In the present study, the physical mechanisms dictating bubble deviation from a spherical shape are investigated by quantifying the magnitude of the bubble's spherical tendencies as the bubble's degree of sphericity. To this end, a numerical treatment of the capillary equation is validated to vapour bubble growth for low Bond numbers. Furthermore, bubble shape evolution is investigated in terms of the Bond number in which the nucleation site radius is the characteristic length.

\section{MATHEMATICAL MODEL}

\section{A. Young-Laplace equation}

Bubble profiles are generated with a numerical treatment of the capillary equation yielding the bubble contour of an individual bubble during quasi-static growth. The capillary equation represents the pressure balance at a point along the bubble contour; this pressure balance is dictated in quasistatic bubble formations by the Young-Laplace equation.

In considering an infinitesimal interfacial segment on a curved surface to be expanding outwards - such as the interface of a growing bubble - the perpendicular segment lengths have associated principal radii of curvature $R_{1}$ and $R_{2}$. Equating the work done by increasing the surface area relative to the surface tension $\sigma$ with the interfacial pressure (often referred to as the capillary pressure), a work balance is established from which the pressure difference at a position $z$ is isolated yielding the Young-Laplace equation (e.g., see Ref. 26),

$$
P_{g}(z)-P_{l}(z)=\sigma\left(\frac{1}{R_{1}(z)}+\frac{1}{R_{2}(z)}\right),
$$

in which $P_{g}$ and $P_{l}$ denote the gas and liquid pressures, respectively.

\section{B. Capillary equation}

In the present investigation, the viscous term of the Young-Laplace equation is not included due to the quasi-static assumption. The capillary equation is developed here with a similar approach as to Refs. 24 and 25 in which the origin of the coordinate system is situated at the bubble apex such that the $z$-axis is oriented downward as illustrated in Fig. 1. 

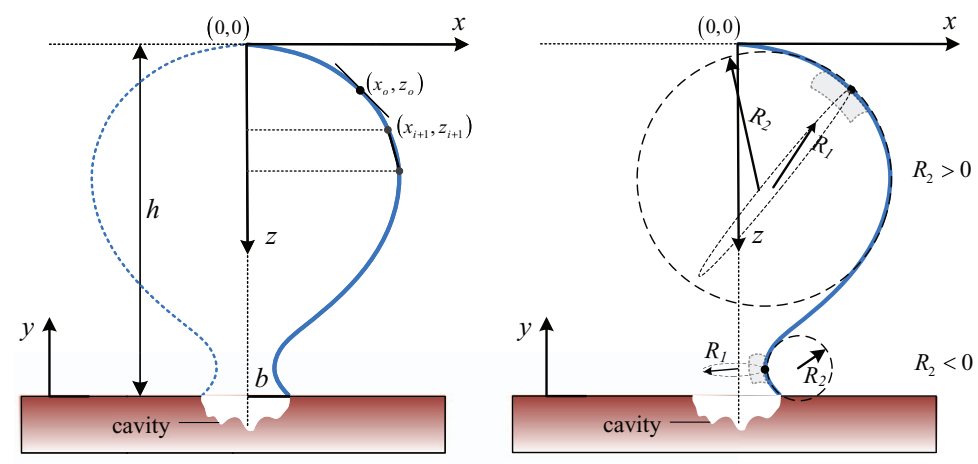

FIG. 1. (Left) Coordinate system for numerical solution to the capillary equation. (Right) Illustration of the principal radii of curvature at different locations along the bubble profile.

By including the hydrostatic pressures, the left-hand side of Eq. (1) is expressed in terms of the vapour and liquid pressures at the apex origin such that

$$
P_{g}(z)-P_{l}(z)=P_{g}(0)-P_{l}(0)-\left(\rho_{l}-\rho_{g}\right) g z .
$$

The symmetry of the problem implies that the principal radii of curvature are equal at the apex origin, noted $R_{o}=R_{1}(0)=R_{2}(0)$. From Eq. (1), the pressure difference at the origin is

$$
P_{g}(0)-P_{l}(0)=\frac{2 \sigma}{R_{o}}
$$

Equations (2) and (3) reduce Eq. (1) to the following form of the capillary equation:

$$
\frac{\sigma}{R_{1}(z)}+\frac{\sigma}{R_{2}(z)}=\frac{2 \sigma}{R_{o}}-\left(\rho_{l}-\rho_{g}\right) g z .
$$

The capillary equation is normalized with the orifice radius $b$ as the characteristic length, such that $x^{*}=x / b$. This exposes the Bond number in the last term,

$$
\frac{1}{R_{1}^{*}}+\frac{1}{R_{2}^{*}}=\frac{2}{R_{o}^{*}}-B o_{b} z^{*} .
$$

The Bond number is conveniently expressed in terms of the cavity radius $b$ and the capillary length, $L_{l}=\sqrt{\sigma /\left(\rho_{l}-\rho_{g}\right) g}$,

$$
B o_{b}=\frac{\left(\rho_{l}-\rho_{g}\right) g b^{2}}{\sigma}=\left(\frac{b}{L_{l}}\right)^{2} .
$$

The capillary equation is deemed valid at any point on the contour of a bubble resulting from quasi-static bubble formation in a quiescent liquid. It is important to note that the last term on the right-hand side of Eq. (5) becomes less significant close to the apex origin. This results in a more spherical shape at the apex origin due to converging principal radii of curvature. Since the magnitude of this term depends on the Bond number, a relatively small Bond number implies a more spherical shape and a relatively large Bond number implies a more oblique bubble shape. Furthermore, this term becomes more significant near the base of the bubble causing it to deviate from spherical. In this way, Eq. (5) suggests that it is the ratio of the cavity radius to capillary length, which dictates bubble shape. As an illustrative example, a small cavity with working conditions generating a small capillary length (due to perhaps a strong gravitational field) will produce bubbles of similar shape to a relatively large cavity with working conditions generating a relatively large capillary length. 


\section{Mode A bubble growth}

For any liquid, gas, and substrate combination at equilibrium, a surface tension balance exists that is dependent on the liquid's affinity to the solid. This phenomenon is known as the wettability of the liquid for a substrate and is quantified by the Young contact angle. ${ }^{2}$ In the case of vapour bubble growth issuing from an orifice, there are two modes of growth possible relative to the contact angle: Mode A and Mode B. ${ }^{25}$

In Mode A, the Young contact angle (liquid side contact angle of a liquid droplet on a horizontal surface at equilibrium) remains inferior to the instantaneous contact angle throughout the growth of the bubble. This is attributed to the working conditions including the liquids affinity for the surface, the nucleation cavity, and the very localised heating source. The result is a vapour bubble emerging from the cavity with a decreasing contact angle as the bubble grows. The contact angle reaches a minimum that is still greater than the Young contact angle thus preventing the foot to expand and then increases until detachment. This effectively means that the triple contact line remains at the perimeter of the nucleation cavity. It is duly noted that, in the case of classical pool boiling applications, this mode is seldom observed.

Conversely, in Mode B, the liquid has a weak affinity for the surface and it yields to the gas bubble's expansion. In this mode, during the bubble growth cycle, the instantaneous contact angle equals the Young contact angle and the bubble foot expands to a radius greater than that of the orifice.

In the present study, Mode A bubble growth is considered. The bubble contact with the perimeter of the nucleation site provides a necessary boundary condition for the numerical treatment of the capillary equation. For this reason, the benchmark experiments use liquids and substrates in which the wettability is such that the bubble foot remains fixed to the orifice. It is duly noted that the capillary equation can be resolved numerically in Mode B as well. In Mode B, the Young contact angle provides the necessary boundary condition. ${ }^{25}$

\section{Numerical methodology}

A numerical treatment of the capillary equation solves Eq. (5) generating a bubble profile. To this end, the radii of curvature are expressed in terms of $x$ and $z, R_{1}=\left(1+z^{\prime 2}\right)^{3 / 2} / z^{\prime \prime}$ and $R_{2}=x(1$ $\left.+z^{\prime 2}\right)^{1 / 2} / z^{\prime},{ }^{25}$ reducing Eq. (5) to

$$
\frac{z^{*^{\prime \prime}}}{\left(1+z^{*^{\prime 2}}\right)^{3 / 2}}+\frac{z^{*^{\prime}}}{x^{*}\left(1+z^{*^{2}}\right)^{1 / 2}}=\frac{2}{R_{o}^{*}}-B o_{b} z^{*} .
$$

Equation (7) solves for $z$ as a function of $x$, which produces an error beyond a certain point along the contour since $z$ is not a proper function of $x$. For this reason, Eq. (7) is also expressed as a function of $z$ by noting that $z^{\prime}(x)=1 / x^{\prime}(z)$ and $z^{\prime \prime}(x)=-x^{\prime \prime}(z) /\left(x^{\prime}(z)\right)^{3}$ yield

$$
\frac{1}{x^{*}\left(1+x^{*^{2}}\right)^{1 / 2}}-\frac{x^{*^{\prime \prime}}}{\left(1+x^{*^{2}}\right)^{3 / 2}}=\frac{2}{R_{o}^{*}}-B o_{b} z^{*} .
$$

Equation (8) is solved numerically assuming that the bubble foot is fixed to the perimeter of the cavity from which the bubble is issuing. It is important to note that the limit of $x(z)$ as $z$ tends to zero does not exist and as such an error is produced at the apex origin when solving Eq. (8). However, the apex origin provides a necessary boundary condition. Therefore, in order to generate a complete profile of the bubble, it is necessary to first solve Eq. (7) to a given point and then solve Eq. (8) for the remaining portion of the profile. The connection between the two equations in the procedure is explained in the following discussion.

Equation (7) is solved using the boundary conditions $z(0)=0$ and $z^{\prime}(0)=0$ at the apex origin. The initial conditions of Eq. (7) require a bubble height, noted $h$ and an arbitrarily chosen apex origin principle radius of curvature $\left(R_{o}\right)$ that is of the order of the cavity radius. With this, Eq. (7) solves for $z$ by interpolation up until the point on the bubble profile in which $z^{\prime}=1$ thereby identifying the coordinates of the point $\left(x_{o}, z_{o}\right)$ illustrated in Fig. 1. Equation (8) then solves for $x$ by interpolation 
with the boundary conditions,

$$
x\left(z_{o}\right)=x_{o}, \quad x^{\prime}\left(z_{o}\right)=1 .
$$

When solving Eq. (8), the same values of $R_{o}$ and $h$ as those chosen for Eq. (7) are used generating a complete bubble contour from $z=0$ to $z=h$.

Recalling that the value of the initial $R_{o}$ is chosen arbitrarily, the resultant bubble profile from the above described procedure is not necessarily in contact with the cavity perimeter. This is illustrated in Fig. 2 in which $y=h-z$. The numerical treatment therefore proceeds by iteration of $R_{o}$ values until the bubble interface is in contact with the perimeter of the cavity (Mode A bubble growth) satisfying the following equality:

$$
x^{*}(h)=1 .
$$

\section{E. Detachment}

At any given moment during the bubble growth cycle prior to detachment, the bubble is considered to be at a height $h$ which is less than or equal to the detachment height noted $h_{d}$. This value of $h$ provides a necessary input value for the numerical procedure. The value of $h$ relative to $h_{d}$ identifies the growth progression of the bubble. In this way, the growth cycle of the bubble is a height scale rather than a time scale. The corresponding numerically generated bubble profiles have a minimum foot radius that is less than the cavity radius. This corresponds to a principal radius of curvature at the apex origin that is too small for the desired normalized foot radius of unity - required in Eq. (10) - and as such incremental increases in $R_{o}$ yield the profile for which the foot is in contact with the perimeter of the cavity. However, at a certain height, noted $h_{d}$, the minimum bubble foot radius is equal to the cavity radius and no such length-steps are required. This profile - illustrated in Fig. 2 - is considered the detachment profile (e.g., see Ref. 25) since any bubble heights greater than $h_{d}$ will yield profiles with a minimum normalized foot radius greater than unity implying that the condition expressed in Eq. (10) cannot be satisfied.

The phenomenon observed in Fig. 2 can be attributed to the fact that the resulting force from the surface tension and adhesion forces manifest at the triple contact line is dependent on the perimeter of the foot of the bubble for Mode A bubble formations. The minimum foot radius corresponds to the minimum surface tension necessary to oppose the buoyancy force in order to keep the bubble
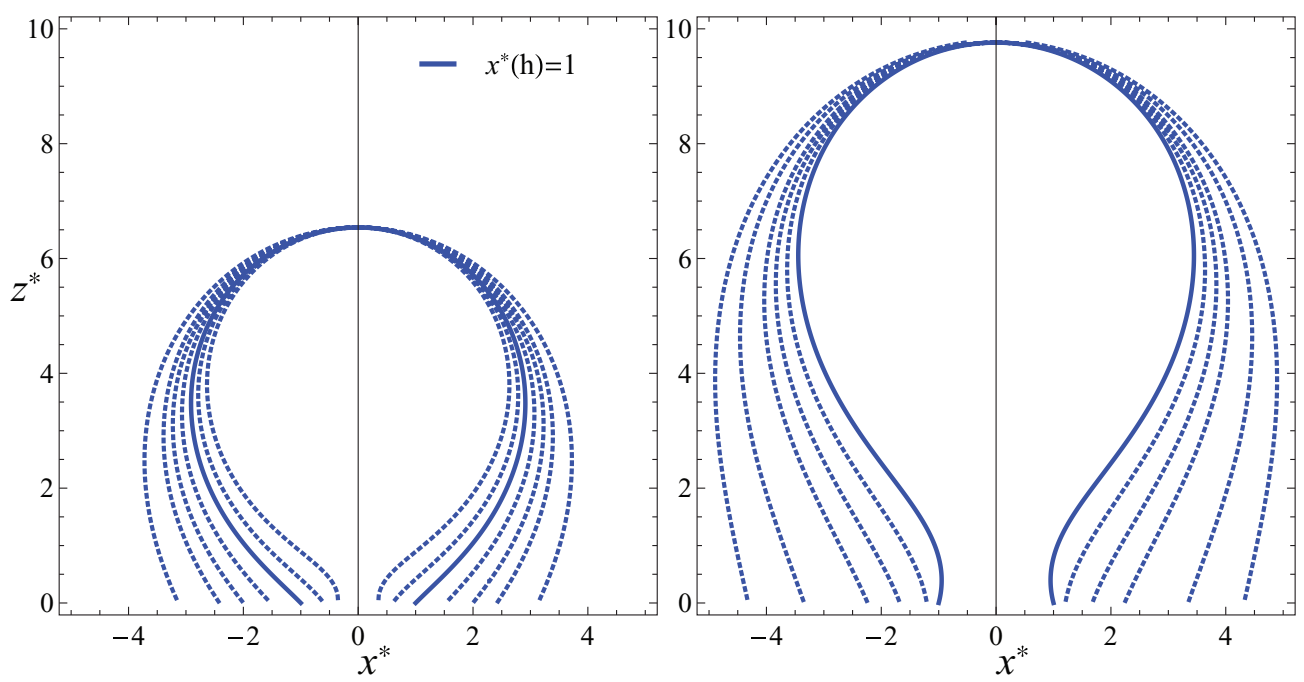

FIG. 2. (Left) Bubble profiles generated by the numerical procedure in which the minimum foot radius is less than unity, $x^{*}(h)<1$. (Right) Bubble profiles generated by the numerical procedure in which the minimum foot radius is equal to unity yielding the profile of the detaching bubble. 
from detaching. The detachment profile therefore corresponds to a bubble that is sufficiently shaped for its buoyancy to necessitate an opposing surface tension requiring a minimum perimeter equal to that of the cavity perimeter. However, this phenomenon is shape dependent in terms of the Bond number as highlighted in Eq. (5).

\section{F. Limitations}

In the present work, the capillary equation's applicability to bubble growth is limited to applications in which the right-hand side of Eq. (4) is greater than or equal to zero. This is due to the fact that a depth $z$ on the profile producing a negative value would invert the Laplace pressure drop $-\sigma\left(1 / R_{1}(z)+1 / R_{2}(z)\right)$ - at that position. Indeed, this could be considered a detachment criterion since the Laplace pressure drop in having changed sign would then be assisting in detachment; however, such considerations are left to future investigations. This study is limited to Bond numbers in which the right-hand side of Eq. (4) is positive for all $z$ values in which $z$ ranges from 0 to $h$. Numerical simulations find this Bond number to be 0.9941 and as such the current investigation considers working conditions yielding Bond numbers that satisfy $B o_{b} \leq 0.9941$.

\section{EXPERIMENTAL SETUP}

In order to validate the capillary equation to vapour bubble growth, an uncomplicated apparatus is built in which a single right angle needle tip provides the nucleation site for bubble growth. The system is designed to capture cross-sectional bubble images throughout the growth cycle. The needle is heated with an electric current inside a basin of pre-boiled distilled water. In an effort to favour bubble growth in which the triple contact line remains fixed to the perimeter of the nucleation site corresponding to Mode A bubble growth, BD Insyte Autoguard needles are used. These needles are made of $B D$ Vialon $^{\mathrm{TM}}$ biomaterial, which is known for its ultra-smooth microsurface in medical applications. Each needle is precision cut with a Sodick AQ300L electroerosion machine cutter ensuring that the needle tip is perpendicular to its staff. The opposite end of the needle is pinched shut to prevent flow through the needle. The interior and exterior diameters of the needles are measured using a Griffin linear microscope with a Mitoyo Absolute Model ID-C112E sliding gauge to an accuracy of $\pm 0.002 \mathrm{~mm}$. The needle tip providing the nucleation site is exposed to its surrounding, while the rest of the needle is wrapped in several layers of Heat-shrink tubing. An electric current heats the needle using a Nautilus Motomaster 10-3490-0 battery capable of 20 A. Electric current intensity is measured using a Metex $M-3800$ multimeter and is controlled using a carbon plate Sargent-welch 530785-10-5 rheostat. In order to favour quasi-static vapour bubble growth, the water temperature is maintained between $96.6^{\circ} \mathrm{C}$ and $98.7^{\circ} \mathrm{C}$ using four evenly spaced $10 \Omega$ resistors. Each resistor is heated with a direct 2 A current supplied by a Welch Scientific Company filtered power supply. The current to the resistors is interrupted during bubble image capturing in order to minimize natural convection flow. The water temperature is measured using an Omega RDXL45D digital thermometer. A Casio EXILIM HS EX-ZRIOO high speed camera is used to capture the quasistatic bubble images. The experiments are repeated three times using three different needles with inner and outer diameters given in Table I. The needle orifice measurement uncertainty is calculated by adding the standard deviation of five measurements with the instrument uncertainty.

The needle is mounted onto an aluminum cylinder resting at the bottom of the basin. The experimental set-up is illustrated in Fig. 3.

TABLE I. Orifice size measurements.

\begin{tabular}{lccc}
\hline \hline Needle & Outer diameter $(\mathrm{mm})$ & Inner diameter $(\mathrm{mm})$ & Uncertainty $(\mathrm{mm})$ \\
\hline $\mathrm{N} 1$ & 0.821 & 0.543 & 0.007 \\
$\mathrm{~N} 2$ & 1.248 & 0.853 & 0.007 \\
$\mathrm{~N} 3$ & 1.666 & 1.185 & 0.004 \\
\hline \hline
\end{tabular}




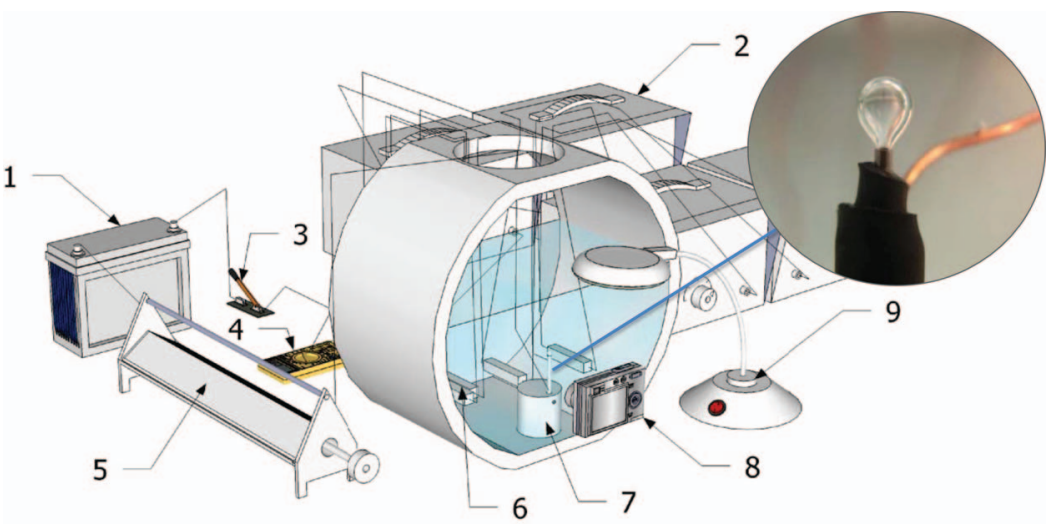

FIG. 3. Schematic representation of the experimental set-up: (1) Battery; (2) power supply; (3) switch; (4) multimeter; (5) rheostat; (6) resistors; (7) needle and support; (8) camera; and (9) lamp.

In an effort to better visualize the shape evolution quantified in terms of the Bond number in Eq. (5), three bubble formation sequences depicting bubble growth for three different Bond numbers are illustrated in Fig. 4. The images show bubbles transitioning from a truncated spherical shape to spherical with an elongation due to neck formation. They show a smaller Bond number with a more spherical bubble evolution than the larger Bond numbers. Also, it is observed that a bubble deviates from a spherical shape near detachment and that this deviation is more prominent for the larger Bond number applications. This suggests that a truncated spherical geometry elongated due to neck formation is more appropriate for bubble growth modeling then a spherical assumption. In the present study, this shape evolution phenomenon is investigated in terms of the Bond number with characteristic length equal to the orifice radius.

\section{VALIDATING THE NUMERICAL PROCEDURE}

Vapour bubble profiles resulting from the numerical treatment of the capillary equation are validated to three test cases corresponding to the nucleation sites N1, N2, and N3. The working conditions are described in Table II and the results are illustrated in Figs. 5-7.

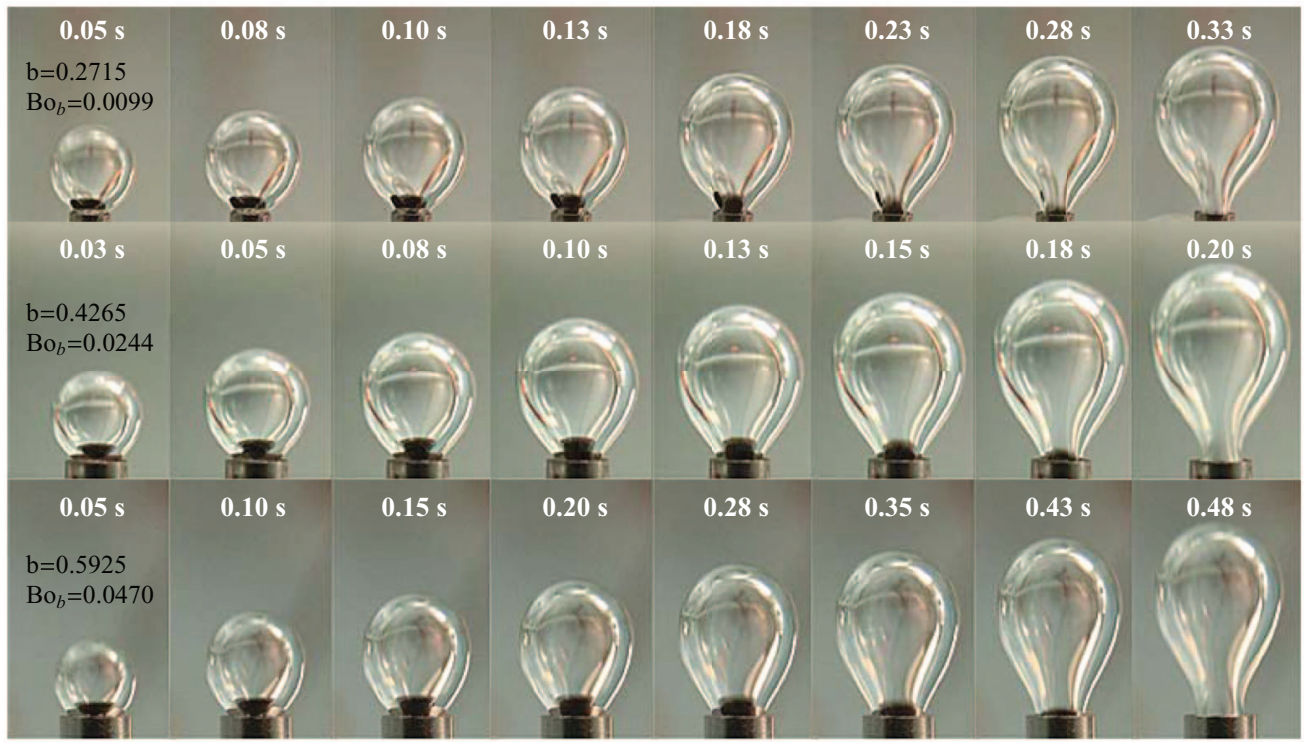

FIG. 4. Visual comparison of vapour bubble images from nucleation sites N1, N2, and N3. The time elapsed since nucleation is indicated at the top of each frame. 
TABLE II. Working conditions for three test cases.

\begin{tabular}{lccc}
\hline \hline Orifice & $\mathrm{Bo}_{b}$ & Water $\left({ }^{\circ} \mathrm{C}\right)$ & Current (A) \\
\hline N1 & 0.0099 & 98.7 & 3.50 \\
N2 & 0.0244 & 97.6 & 4.48 \\
N3 & 0.0470 & 96.6 & 5.01 \\
\hline \hline
\end{tabular}

For the growth profiles presented in these figures, the height of the experimentally measured bubbles is used as the input value for their corresponding numerically generated profiles. For the detachment profiles, the numerical contours are generated using the treatment described in Sec. II E and do not require a height input value, since the detachment height can be numerically generated. In an effort to validate this method, the numerically generated detachment profile is compared
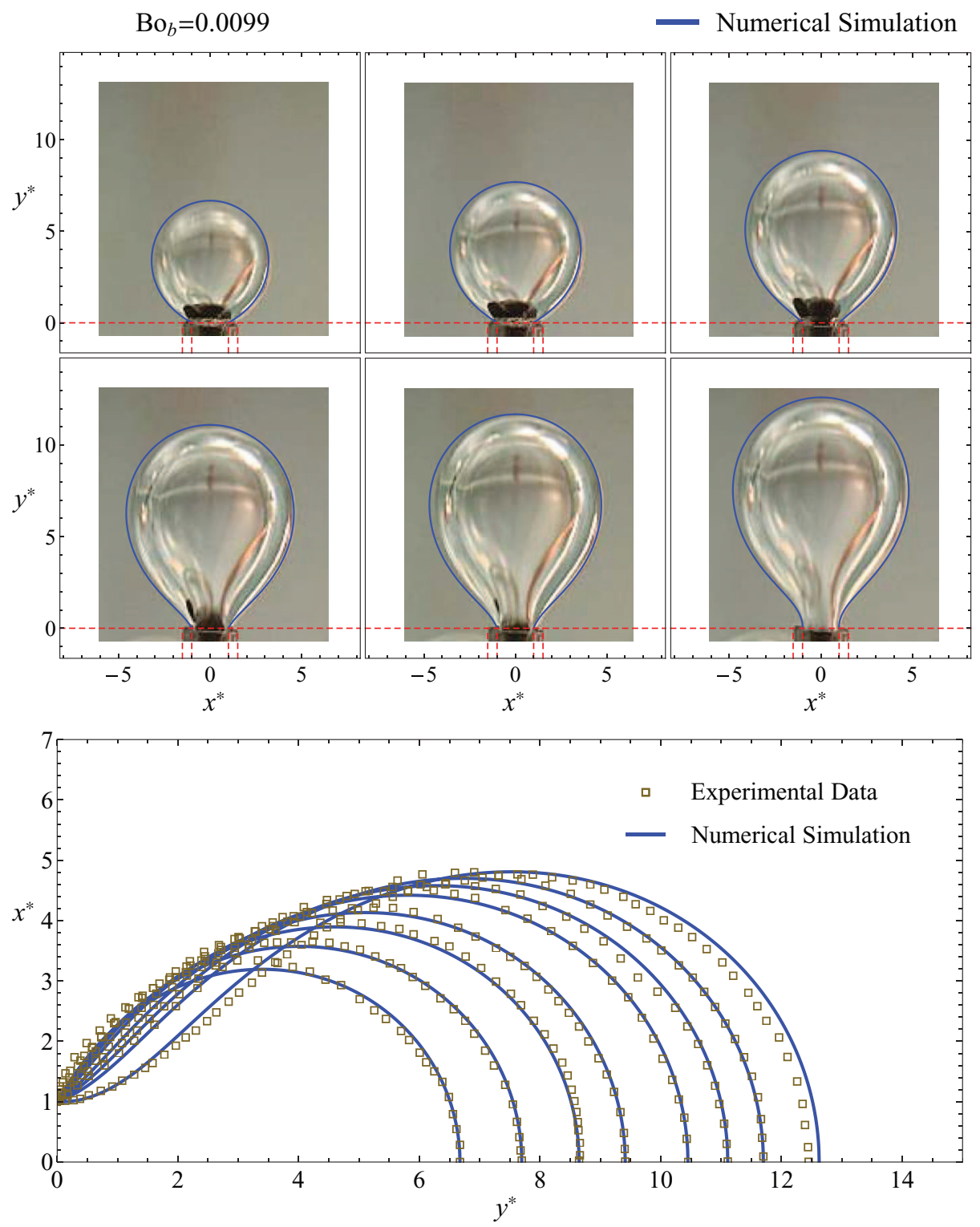

FIG. 5. Bubble profiles during bubble evolution as predicted by the solution of the capillary equation compared with bubble growth due to vaporization profiles from a nucleation site of inner diameter $0.543 \mathrm{~mm}$. 

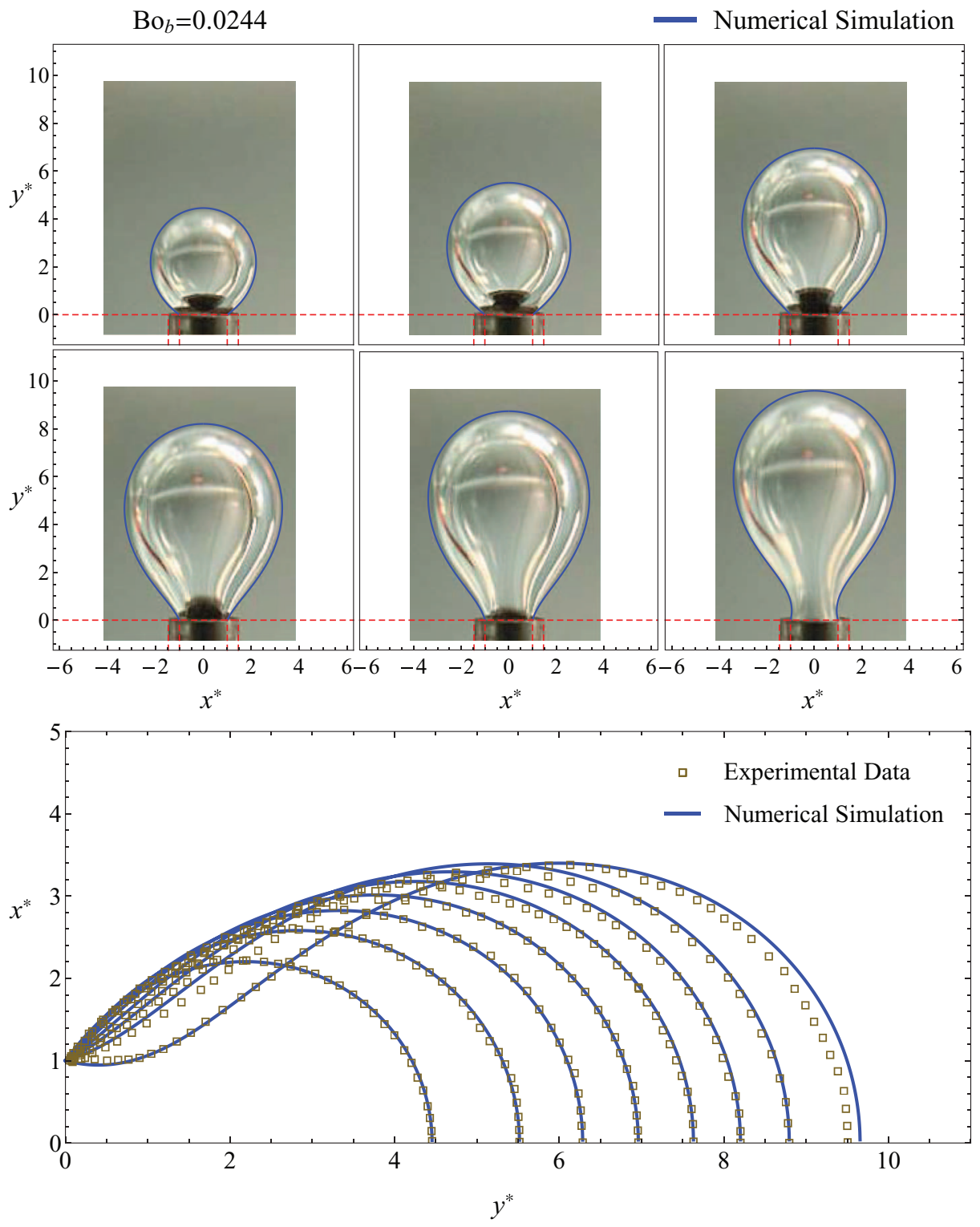

FIG. 6. Bubble profiles during bubble evolution as predicted by the solution of the capillary equation compared with bubble growth due to vaporization profiles from a nucleation site of inner diameter $0.853 \mathrm{~mm}$.

with the last captured image prior to the dynamic phenomenon known as bubble pinch-off. It is carefully noted that the bubble growth treatment in this document is quasi-static and for this reason bubble pinch-off and bubble detachment are considered simultaneous events. Indeed, the beginning of pinch-off is often equated with the moment of bubble detachment since the life of pinch-off compared with the growth cycle of the bubble makes deformation of the neck and detachment of the bubble nearly simultaneous events. ${ }^{27}$ Experimental and numerical results illustrating that bubble pinch-off is a dynamic process and that it is only a fraction of the necking phenomenon are detailed in Refs. 28-32.

As previously discussed, the bubble profile resulting from the numerical treatment of the capillary equation has been validated for gas diffusion and gas injection bubble formation scenarios by Refs. 24 and 25, respectively. The present investigation builds on these works by testing the ability of the capillary equation to accurately predict the bubble profiles of bubble growth due to 

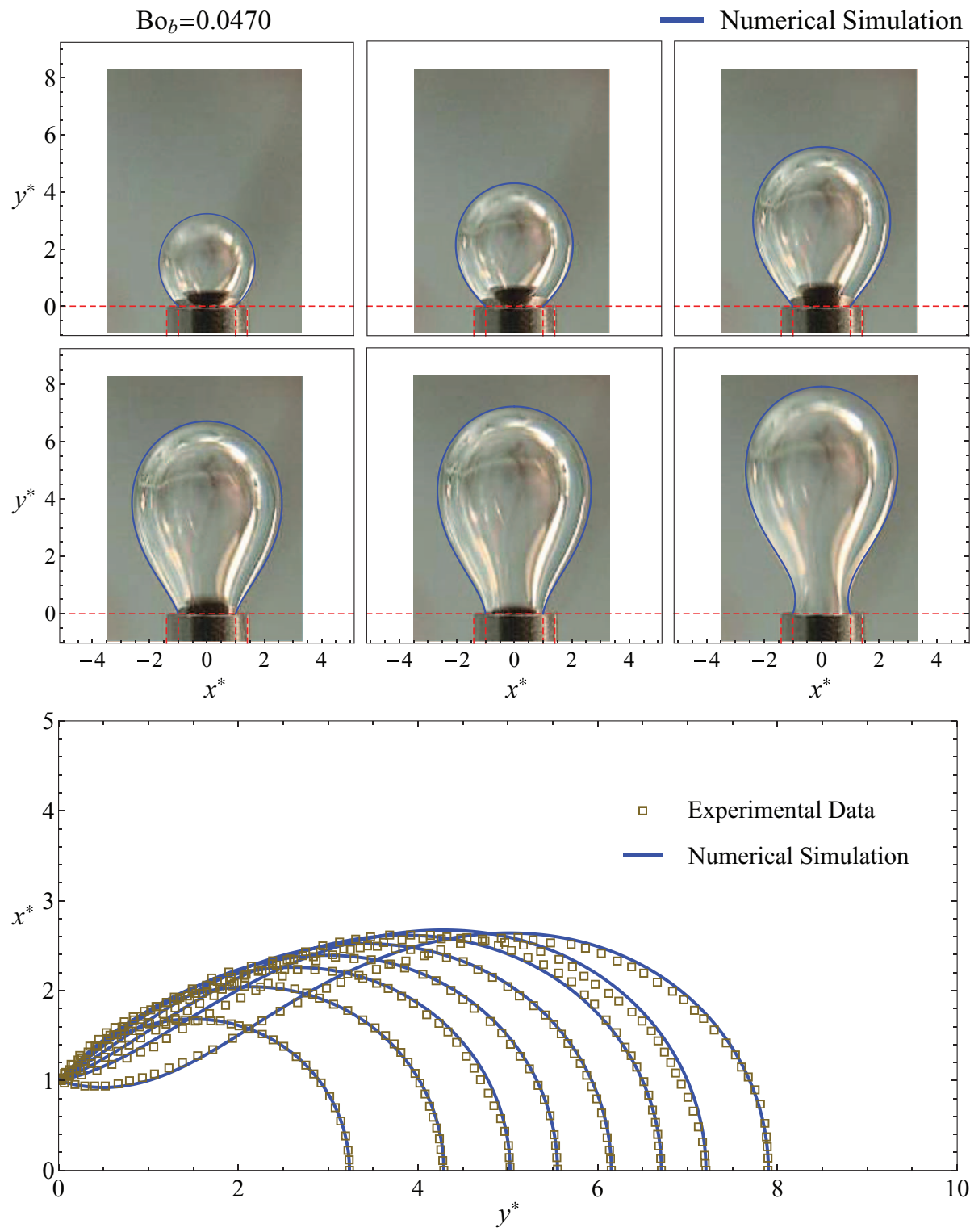

FIG. 7. Bubble profiles during bubble evolution as predicted by the solution of the capillary equation compared with bubble growth due to vaporization profiles from a nucleation site of inner diameter $1.185 \mathrm{~mm}$.

vaporization. Figure 5 compares the bubble profiles resulting from the numerical treatment with the experimental results in which the \pm 3 pixel uncertainty in the measured profiles from the captured images is represented by the height and width of the data plot markers.

The bubble profiles from the captured images of the $0.853 \mathrm{~mm}$ inner diameter nucleation cavity are measured and compared with the profiles generated by the numerical solution to the capillary equation. The results are illustrated in Fig. 6.

From the larger nucleation site N2 relative to N1, the bubble is observed to be less spherical throughout its growth cycle. The largest orifice used for vapour bubble growth in this study is of inner diameter $1.185 \mathrm{~mm}$. The bubble profiles from the captured images during bubble growth and at detachment are measured and compared with those of the numerical solution to the capillary equation and are illustrated in Fig. 7 below. 

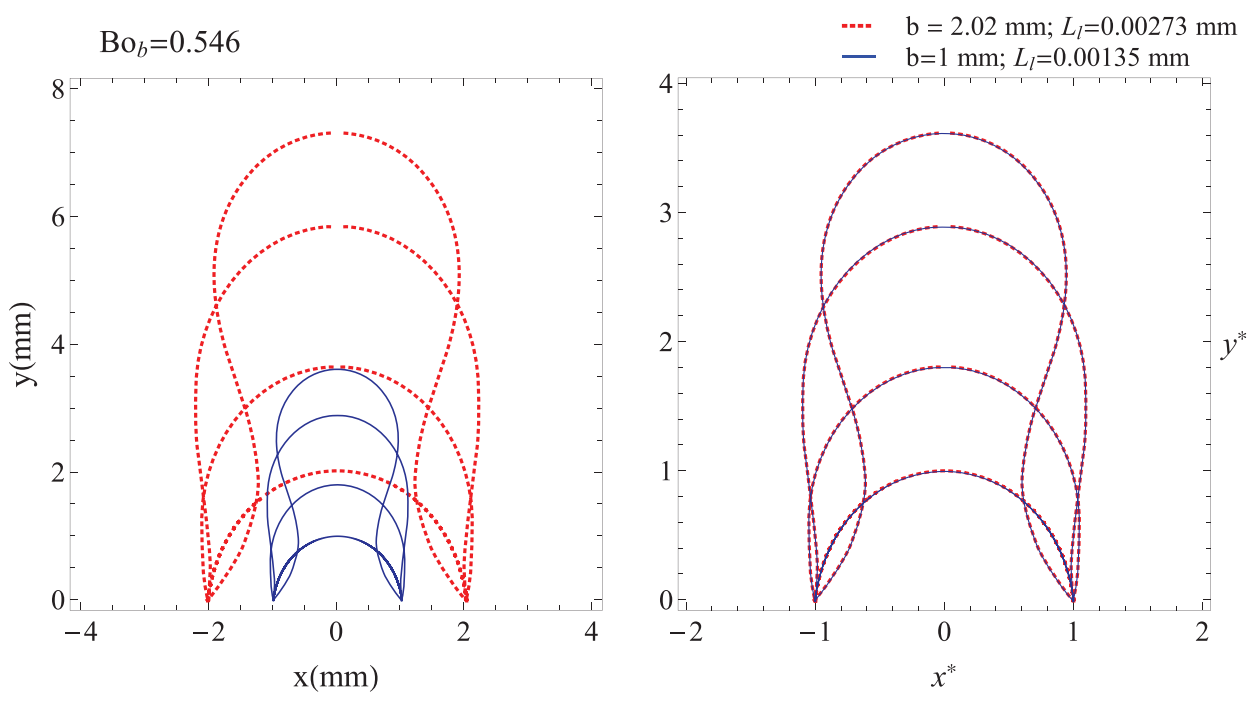

FIG. 8. Comparison of numerically generated bubble profiles of different sizes with the same Bond number 0.546: (left) size comparison; (right) shape comparison.

\section{RESULTS OF NUMERICAL ANALYSIS}

\section{A. Bubble shape}

With the numerical treatment validated, the shape evolution of quasi-static vapour bubble growth is investigated. In particular, the shape of the numerically generated bubble profiles is shown to be dependent on the Bond number. To illustrate this, profiles are normalized by the cavity radius showing that the same Bond number generates bubble growth of the same shape yet not necessarily of the same size. This shows that the bubble is dependent on the Bond number for its shape and on the cavity radius for its size as suggested by Eq. (5). Figure 8 compares the numerically generated profiles for two bubbles with the same Bond number yet under different working conditions. One simulation is of a vapour bubble issuing from a cavity of $2.02 \mathrm{~mm}$ radius for working conditions yielding a capillary length of $0.00273 \mathrm{~mm}$ (e.g., water under terrestrial gravity conditions). The other simulation depicted in Fig. 8 is of a vapour bubble issuing from a cavity of $1 \mathrm{~mm}$ radius with working conditions yielding a capillary length of $0.00135 \mathrm{~mm}$ (e.g., water under a gravitational field strength of $40 \mathrm{~m} / \mathrm{s}^{2}$ ). Both of these scenarios yield a Bond number of 0.546 and as such generate bubbles of identical shape.

The similar shapes for the two different sized bubbles illustrated in Fig. 8 are attributed to the hydrostatic pressures of equal relative importance for each simulation applying pressure along the bubble profile which increases with depth. The importance is relative since - for example - the bubble that is larger due to the larger orifice has more depth yet a weakened gravitational field strength, and thus a similar deformation to that of the smaller bubble with a stronger gravitational field strength. Even still, the practical significance is that although the relative shape is the same, more energy would be required to form and detach the bubble of larger volume from the larger nucleation site.

\section{B. Bubble deformation with respect to Bond number}

The influence of the Bond number on the bubble shape can best be illustrated with a nondimensional comparison of bubble profiles for varying Bond numbers. To this end, the bubble profiles of the numerical solution to the capillary equation are normalised by the bubble height such that $\hat{x}=x / h$ for three different Bond numbers: 0.00137, 0.134, and 0.546. In Fig. 9, the bubble contours of the three different Bond numbers are compared at three different moments in the growth cycle: the most hemispherical moment $h=b$, the moment in which the bubble height is 

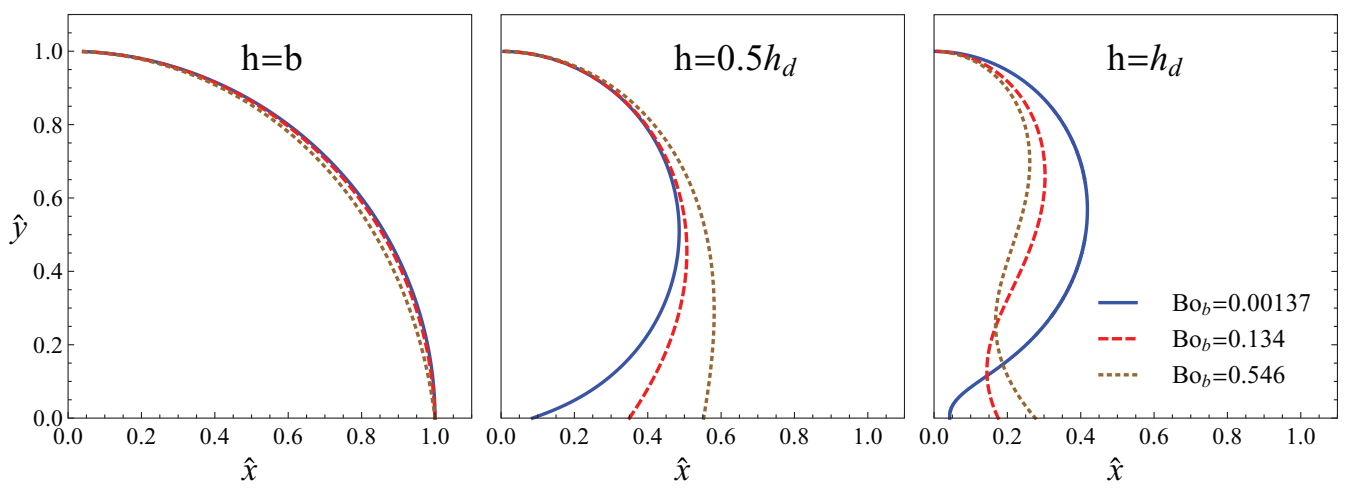

FIG. 9. Comparison of bubble profiles for different Bond numbers.

half that of the detachment height $h=0.5 h_{d}$, and the detachment frame $h=h_{d}$. The results show that a relatively large Bond number generates bubble contours with an inward constraint thereby causing the bubble shape to deviate from a spherical shape and to elongate with neck formation near detachment. Conversely, a small Bond number favours a more spherical bubble shape throughout the growth cycle.

\section{Bubble aspect ratio evolution}

The commonly used Aspect Ratio (AR) of the numerically simulated bubble is investigated. The aspect ratio as defined by Refs. 33 and 34 is simply the ratio of the major axis length to the minor axis length, and is sometimes referred to as the bubble shape factor. ${ }^{35}$ In Figure 10, the bubble AR evolution, quantified in terms of bubble height normalized by its detachment height $h^{+}=h / h_{d}$, resulting from the numerically generated bubble profiles is illustrated for working conditions generating varying Bond numbers. The results show larger AR values near detachment for larger Bond numbers. For smaller Bond numbers, irrespective of bubble size, the bubble AR is closer to unity throughout the bubble growth cycle. Recalling that a perfect sphere has an AR of unity, the upward AR trends illustrated during early growth correspond to the bubble transitioning from hemispherical to spherical. During mid growth, an AR of unity is obtained corresponding to the most spherical shape attained during the bubble growth cycle. From this point onwards to detachment, the AR continues to increase for values greater than unity. This is attributed to bubble

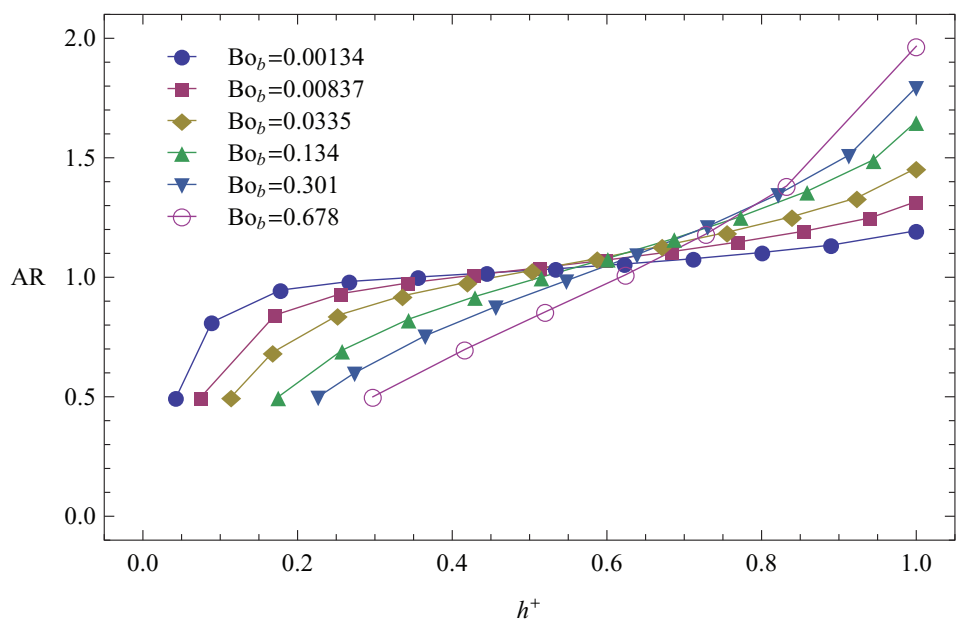

FIG. 10. Bubble aspect ratio evolution from numerical simulations for varying Bond numbers. 
elongation due to the necking phenomenon. The deviation from an AR of unity during the bubble growth cycle is more prominent for larger Bond numbers and is independent of bubble volume.

\section{Bubble degree of sphericity during bubble growth}

A convenient way to quantify the spherical behaviour of a bubble, or conversely its level of deformation, is to evaluate its degree of sphericity. The bubble degree of sphericity, noted $\Psi$, is the ratio of the bubble's volume equivalent spherical area to the bubble's measured surface area,

$$
\Psi=\frac{\left(6 \sqrt{\pi} V_{m}\right)^{2 / 3}}{A_{m}},
$$

in which $V_{m}$ is the bubble's measured volume and $A_{m}$ is the bubble's measured surface area including the bubble foot area projected onto the plane normal to the bubble's vertical axis. The measured volume and surface area are calculated from the numerically generated bubble contour using a conical frustum method. More precisely, since the bubble is symmetric about its vertical axis, a rotation about the central axis generates the volume of the bubble. Since the numerical procedure generates a series of contour coordinate points, the volume is calculated by solving for the following sum of conical volumes:

$$
V_{m}=\frac{1}{3} \sum_{i=1}^{n-1}\left(y_{i+1}-y_{i}\right)\left(x_{i+1}^{2}+x_{i+1} x_{i}+x_{i}^{2}\right) .
$$

A similar conical frustum analysis generates the bubble area,

$$
A_{m}=\pi \sum_{i=1}^{n-1}\left(x_{i+1}+x_{i}\right) \sqrt{\left(x_{i}-x_{i+1}\right)^{2}+\left(y_{i+1}-y_{i}\right)^{2}}+\pi b^{2} .
$$

In Fig. 11, the bubble degree of sphericity evolution resulting from the numerically generated bubble profiles is illustrated for working conditions generating varying Bond numbers. The results show similar trends for all Bond numbers tested. Generally, during early growth, a bubble's degree of sphericity increases towards unity. Recalling that the maximum attainable degree of sphericity is unity corresponding to a perfect sphere, this is attributed to a transitioning phase from hemispherical to spherical. During mid growth, a maximum sphericity is attained representing the bubble's most spherical shape followed by a sharp decrease attributed to neck formation causing the bubble to elongate and become more oblique. The deviations from a sphericity of unity during the mid and late stages of the bubble formation are more pronounced and occur earlier for larger Bond numbers. The shape evolution insight that the degree of sphericity analysis provides is that the maximum attainable degree of sphericity decreases with increasing Bond number.

\section{E. Bubble degree of truncated sphericity}

The results of the bubble degree of sphericity study of Sec. V D show that a spherical bubble shape assumption in bubble growth modeling can be erroneous. In light of this, as a starting point in the development of more appropriate bubble geometric modeling, the validity of a truncated spherical geometric bubble shape with a fixed base radius, as illustrated in Fig. 12, is investigated.

To this end, the bubble degree of truncated sphericity, noted $\Psi_{t r}$, is introduced. It is defined as a ratio of areas. The numerator of the area is of a truncated sphere of equal volume to that of the bubble. The denominator is the area measured from the bubbles profile. It is important to note that the areas include the base of the bubble in the plane normal to its central vertical axis. The bubble degree of sphericity is defined as

$$
\Psi_{t r}=\frac{A_{t r} \circ V_{t r}^{-1}\left(V_{m}\right)}{A_{m}} .
$$




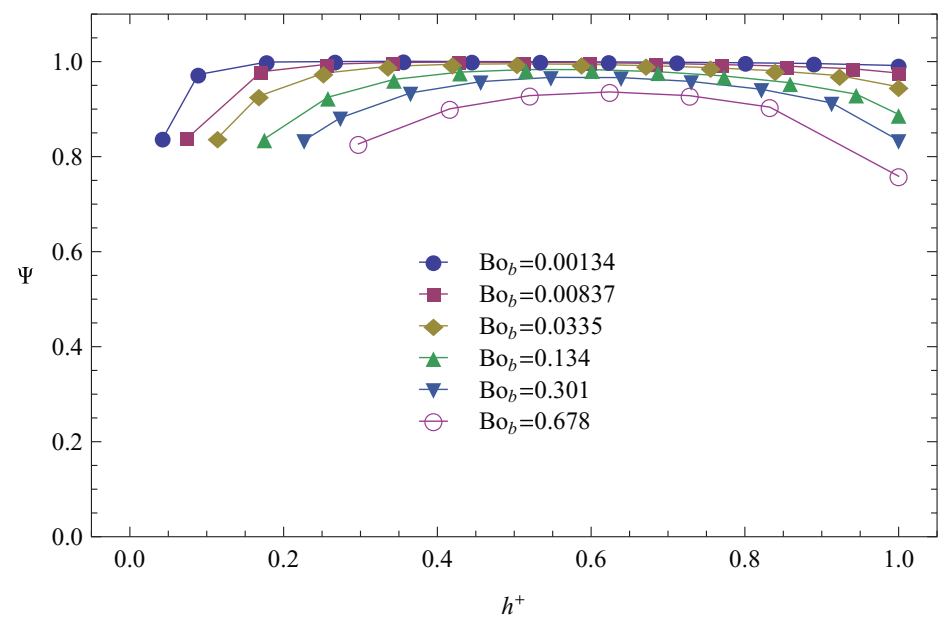

FIG. 11. Bubble degree of sphericity evolution from numerical simulations for varying Bond numbers.

As shown above, the surface area and volume of a truncated spherical segment of radius $R$ atop a cavity of radius $b$ are, respectively,

$$
A_{t r}(R)=2 \pi R\left(R+\sqrt{R^{2}-b^{2}}\right)+\pi b^{2}
$$

and

$$
V_{t r}(R)=\frac{1}{3} \pi\left(2 R-\sqrt{R^{2}-b^{2}}\right)\left(\sqrt{R^{2}-b^{2}}+R\right)^{2} .
$$

It is postulated that, by minimizing the available vapour-liquid surface area, the truncated spherical segment, with base fixed to the perimeter of the cavity from which the bubble emerges, is a more appropriate geometric assumption than a spherical assumption. The accuracy of this postulation is measured in terms of the degree of truncated sphericity in which unity is the validating criterion. Furthermore, the physical mechanism responsible for a deviation from a truncated sphericity of unity is investigated. It is carefully noted that any bubble elongation would yield a bubble degree of truncated sphericity that is less than unity and that any inward deformation from hemispherical would result in a bubble degree of truncated sphericity greater than unity. In Fig. 13, the bubble

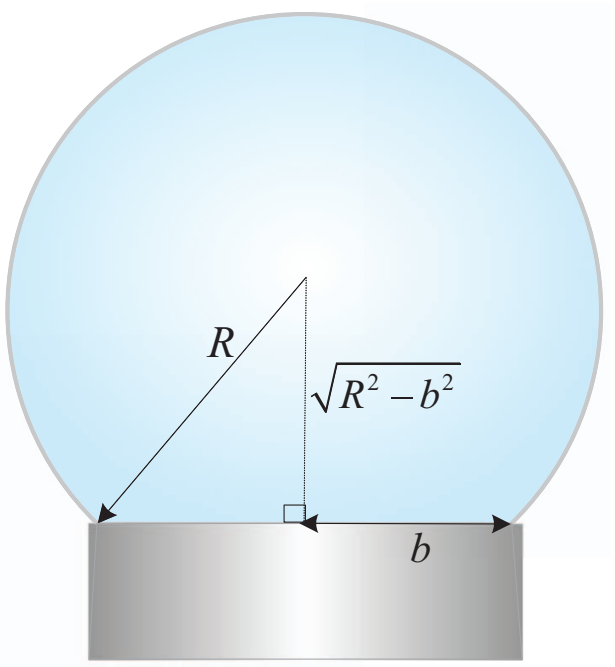

FIG. 12. Truncated spherical bubble of radius $R$ and foot radius equal to the cavity radius. 


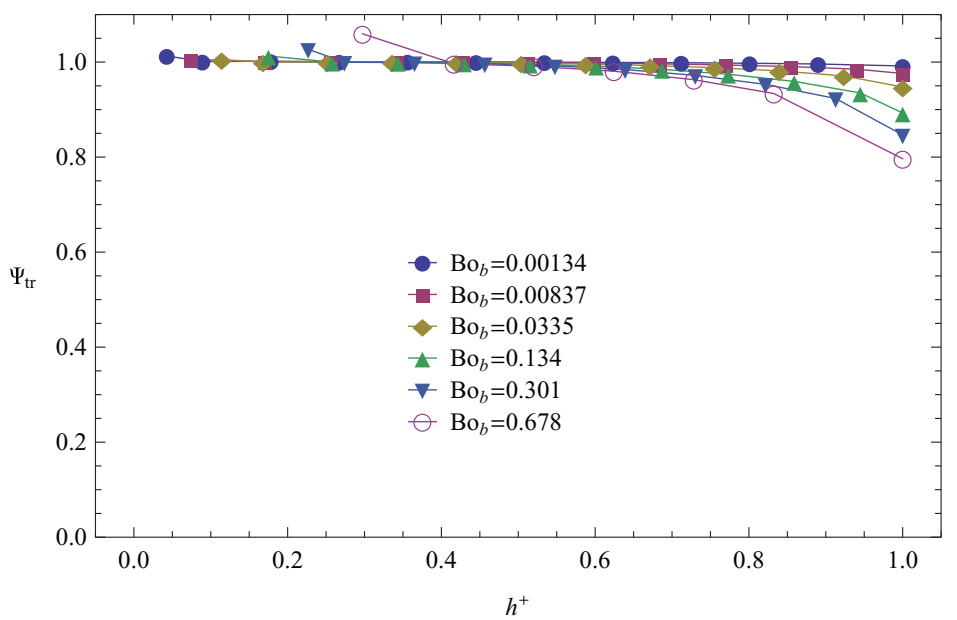

FIG. 13. Bubble degree of truncated sphericity evolution from numerical simulations for varying Bond numbers.

degree of truncated sphericity evolution resulting from the numerically generated bubble profiles is illustrated for working conditions generating varying Bond numbers.

The results show that during early growth the bubble degree of truncated sphericity is near unity for low Bond numbers. For the larger Bond numbers, the hemispherical shape is altered by inward compressions of the bubble outer walls yielding truncated sphericity degrees greater than unity. After attaining unity, during mid growth, the bubble degree of truncated sphericity begins to decrease attributed to bubble elongation due to neck formation. The deviation from a truncated sphericity of unity is shown to be more pronounced and to occur earlier in the growth cycle for larger Bond numbers. Generally, it is shown that in all bubble formations tested, the bubble's degree of truncated sphericity remains closer to unity than the bubble's degree of sphericity throughout the growth cycle. As an illustrative example of this, the profile of a $B o_{b}=0.648$ bubble formation is considered. The volume is measured using Eq. (12). Its volume equivalent truncated spherical radius is solved for using the inverse function of Eq. (16) noted $V_{t r}^{-1}$. The profiles of the resultant volume equivalent truncated sphere and the volume equivalent sphere are compared to the numerically generated profile in Fig. 14.

The significance of this result lies in the fact that the bubble shape predictions can be greatly improved by assuming it to be a growing truncated sphere rather than a growing sphere and that the corresponding size and shape characteristics can still be expressed in simple terms such as the bubble radius - given that the cavity radius is known. Therefore, such characteristics such as bubble detachment radius $R_{d}$ would correspond to a detachment volume equal to $V_{t r}\left(R_{d}\right)$.

\section{F. Bubble deformation due to local stresses}

The following pressure balance analysis investigates the hydrostatic pressure distribution and the capillary pressure distribution over a growing bubble. The capillary equation is numerically solved for different Bond numbers over various stages of the bubble growth cycle. Furthermore, in order to further understand the influence of local stresses on bubble shape, the principal radii of curvature resulting in changes in the pressure distribution are investigated. These are normalized by the radius of curvature at the bubble apex, noted $R_{o}$, such that

$$
\bar{R}_{i}^{-1}=\frac{R_{o}}{R_{i}}
$$

It is important to recall that the capillary equation expressed in Eq. (4) is an interfacial balance of pressures at a depth $z$ from the bubble apex. The left-hand side of Eq. (4) is often referred to as the Laplace pressure drop at depth $z$ and is noted here as $P_{L, z}$. The right-hand side of Eq. (4) is the 


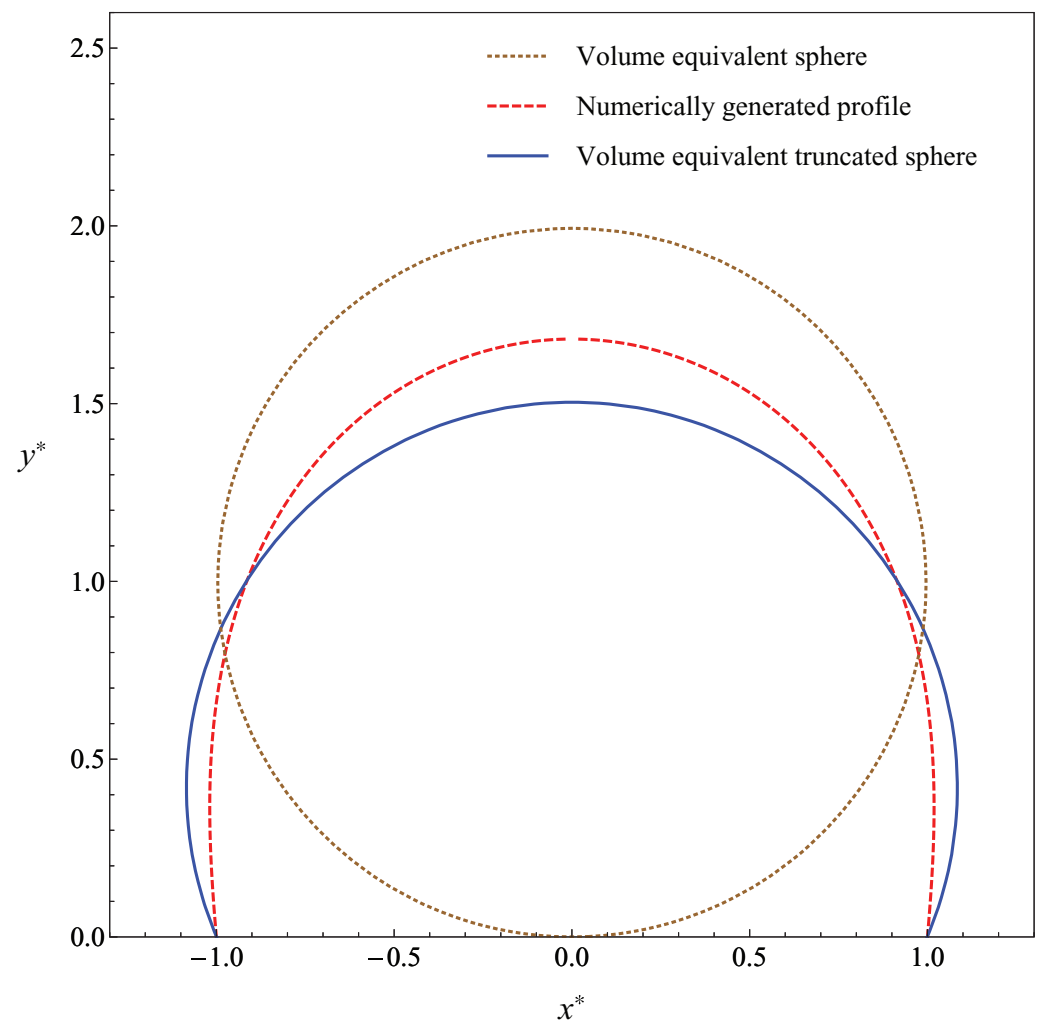

FIG. 14. Numerically generated profile of a $B o_{b}=0.648$ bubble formation at midgrowth compared with the profiles of its volume equivalent sphere and truncated sphere.

difference between the Laplace pressure drop at the apex, noted $P_{L, o}$, and the hydrostatic pressure at depth $z$, noted $P_{h y d, z}$. The capillary equation can therefore be expressed as a balance of pressures at a depth $z$ from the apex of the bubble such that

$$
P_{L, z}=P_{L, o}-P_{h y d, z} .
$$

With depth, the hydrostatic pressure gains importance causing the Laplace pressure drop at position $z$ to diverge from the Laplace pressure drop at the apex origin. The relative importance of the hydrostatic pressure is dependent on the Bond number, as can be seen by the normalized capillary equation expressed in Eq. (5).

In this way, the local stresses acting on the bubble are evaluated and presented in Figs. 15-17. It is shown that the degree to which the hydrostatic pressure causes the Laplace pressure drop at a depth $z$ to deviate from the Laplace pressure drop at the apex is dependent on the Bond number.

Before analysing the results, it is important to recall the physical significance of the principal radii of curvature illustrated in Fig. 1. They represent the curvature in two planes perpendicular to the tangent plane of a given point on the contour. An important feature for bubble growth is that $\bar{R}_{1}^{-1}$ can at no point dissolve to zero since this would imply an infinite bubble width. Furthermore, a change in $\bar{R}_{2}^{-1}$ sign implies a change in concavity. In particular, a negative $\bar{R}_{2}^{-1}$ value implies an outward curvature (relative to the central vertical axis) causing an inward bubble deformation from spherical. Consequentially, $\bar{R}_{2}^{-1}=0$ represents the location of an inflexion point and appears as a straight portion, possibly infinitely short, of the bubble profile and serves to identify the beginning of the necking phenomenon. Therefore, if a negative $\bar{R}_{2}^{-1}$ occurs and is located along the profile away from the foot of the bubble, the location of the minimum negative value of $\bar{R}_{2}^{-1}$ would appear as an inward pinching of the neck of the bubble and would be accompanied by smaller values of $R_{1}$. For this reason, the minimum negative value of $\bar{R}_{2}^{-1}$ attained along the bubble profile, coinciding with the maximum value of $\bar{R}_{1}^{-1}$ attained, identifies the most inward pinch. To summarize, deformation 

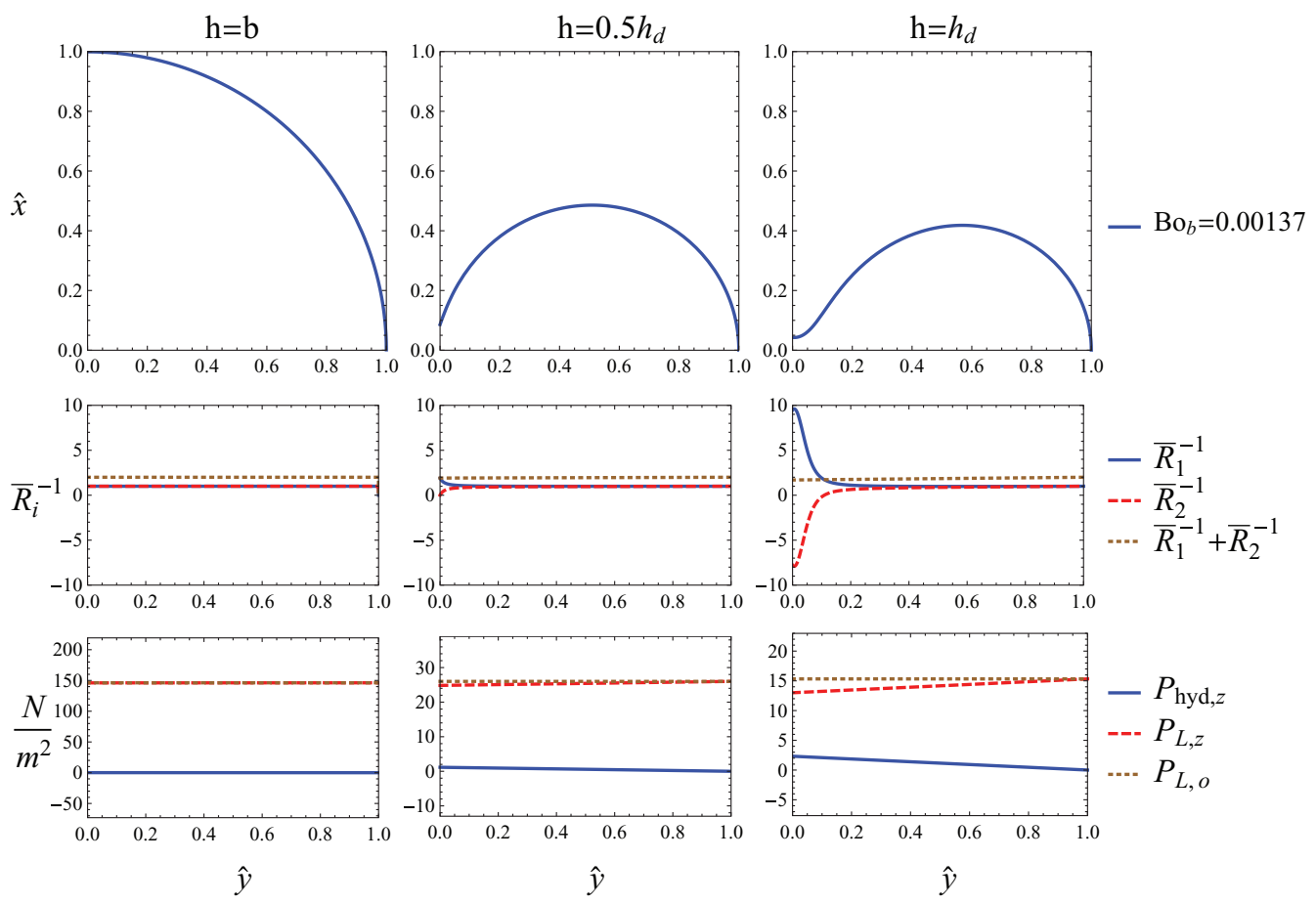

$-P_{\text {hyd }, z}$

--- $P_{L, z}$ $\cdots P_{L, o}$

FIG. 15. Local stresses analysis for bubble growth with Bond number 0.00137.
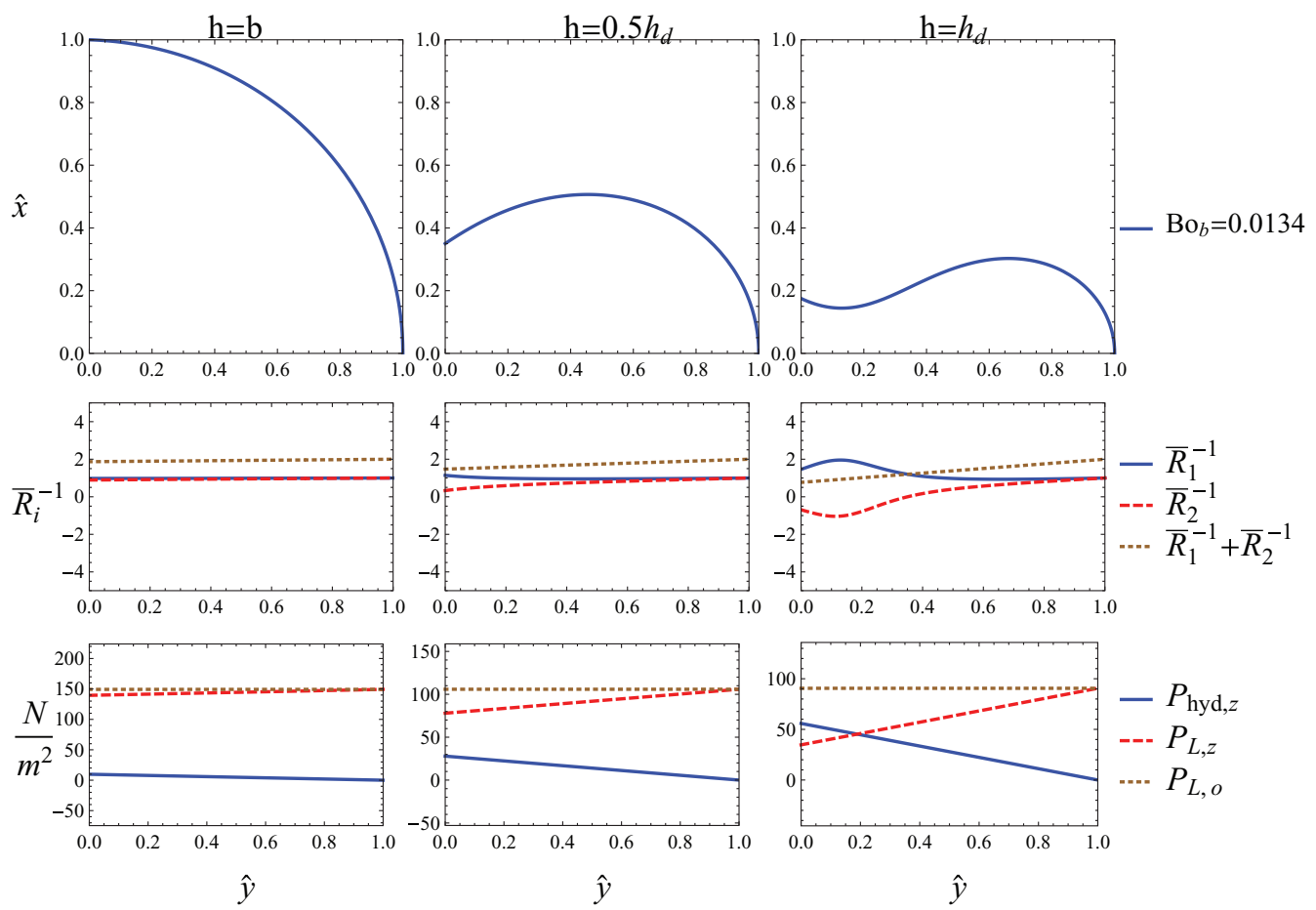

FIG. 16. Local stresses analysis for bubble growth with Bond number 0.134. 

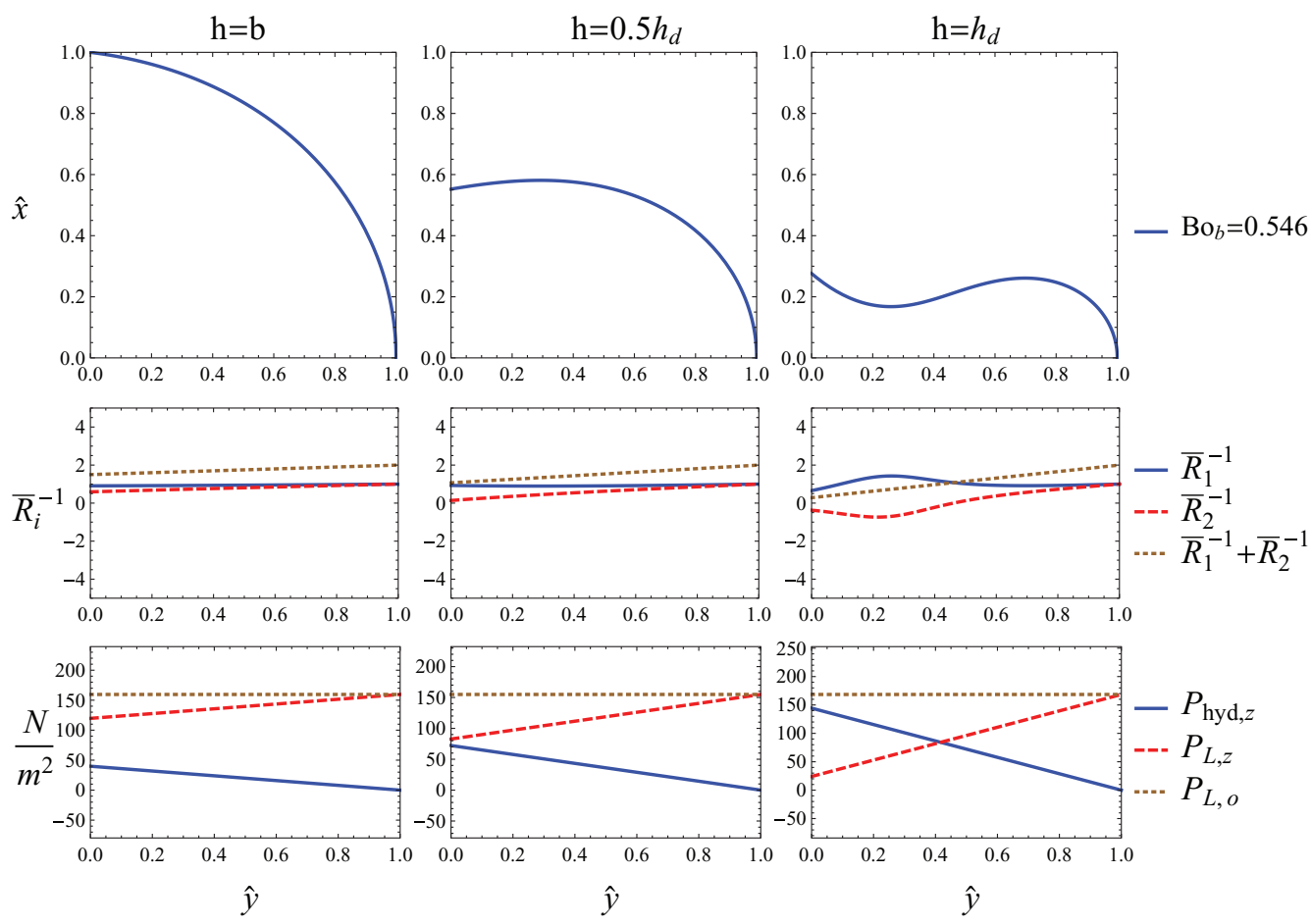

FIG. 17. Local stresses analysis for bubble growth with Bond number 0.546.

from a truncated spherical shape can by identified by the coinciding minimum and maximum values of $\bar{R}_{2}^{-1}$ and $\bar{R}_{1}^{-1}$, respectively, being located away from the foot of the bubble.

Figure 15 illustrates the principal radii of curvature distribution as well as the pressure distribution over a bubble for working conditions yielding a Bond number of 0.00137 . The bubble contour coordinate points are normalized by the bubble height such that $\hat{x}=x / h$. Three different moments in the growth cycle are considered: the most hemispherical moment $h=b$, the moment in which the bubble height is half that of the detachment height $h=0.5 h_{d}$, and the detachment frame $h=h_{d}$.

Figure 16 illustrates the evolution of the pressure distribution over a bubble for working conditions yielding a Bond number of 0.134. It is important to note that for this larger Bond number, the hydrostatic pressure plays a more influential role and as such, bubble deformation from spherical is more significant. Another important feature is that the absolute maximum values of $\bar{R}_{1}^{-1}$ and $\bar{R}_{2}^{-1}$ are not located at the foot of the bubble in the detachment frame. Since these values locate the most inward pinching, their locations along the neck of the bubble imply significant deformation from spherical.

Figure 17 illustrates the evolution of the pressure distribution over a bubble for working conditions yielding a Bond number of 0.546. Once again, in the analysis of this evolution, it is important to note the role of the hydrostatic pressure. In particular, the hydrostatic pressure is more dominant than the Laplace pressure drop term at some depth $z$ for the majority of the bubble growth cycle. As a result, the neck formation is significant in the bubble shape evolution.

The results show that a low Bond number favours a spherical bubble shape throughout the growth cycle. This is demonstrated in Fig. 15 by the two principle radii of curvature being equal to each other over the majority of the bubble contour and the absolute maximums of $\bar{R}_{2}^{-1}$ and $\bar{R}_{1}^{-1}$ being located at the bubble foot implying little deformation due to inward pinching. By contrast, the larger Bond number simulation yields principal radii of curvature that are only equal near the bubble apex implying a less spherical bubble throughout the growth cycle. Furthermore, the maximum value of $\bar{R}_{1}^{-1}$ occurs along the neck of the bubble implying that larger Bond numbers favour deformation in the way of inward pinching relative to the central axis. This is attributed to the observation that, for larger Bond numbers, the hydrostatic pressure at some point during the bubble's evolution dominates 
the Laplace pressure drop at a depth $z$ in the lower section of the bubble causing deformation from spherical. This phenomenon was not observed for the lower Bond bubble formation.

The results of the local stresses analysis correspond well with the observed trends of deviation from a truncated sphericity of unity while providing insight into the mechanism that is responsible for bubble deformation. For example, the slope of $\bar{R}_{2}^{-1}$ with respect to $y$ is positive for the entire bubble profile until the hydrostatic pressure becomes dominant at the time when slope of $\bar{R}_{2}^{-1}$ becomes negative corresponding to an inward pinching. Prior to this occurrence in the growth cycle, the bubble appears to remain a spherical portion with straightening outer walls near the bubble foot. It is therefore postulated here that, prior to the hydrostatic pressure dominating the Laplace pressure drop at some depth $z$, a reasonable bubble shape approximation that would simplify any analytical attempts to solving the bubble growth problem would be to assume the bubble to be a truncated spherical portion rising due to a cylindrical neck at its base. This crucial location in bubble shape transformation in which the hydrostatic pressure becomes dominant can be identified by considering the hydrostatic pressure to be greater than or equal to the Laplace pressure drop at depth $z$ resulting in

$$
R_{o}^{*} z^{*} \geq \frac{1}{B o_{b}}
$$

This result is significant as it illustrates the dependence of bubble shape on the Bond number. Notably, for very small Bond numbers, the product on the left-hand side must be very large to satisfy the inequality making bubble deformation due to hydrostatic pressure dominance less likely away from the bubble foot. When analysing the above inequality, it is useful to consider its significance at the foot of the bubble $(z=h)$. This is due to the fact that, during the growth of the bubble, the hydrostatic pressure first surpasses the Laplace pressure drop at the foot of the bubble. For this reason, the smallest Bond number for which bubble deformation due to a dominant hydrostatic pressure term occurs is solved by equating the inequality of Eq. (19) and evaluating it at the bubble foot yielding

$$
R_{o}^{*}=\frac{1}{B o_{b} h^{*}} .
$$

When including Eq. (20) into the numerical treatment of the capillary equation for a detaching bubble, the capillary equation solution procedure provides one possible Bond number: 0.06032. Therefore, for Bond numbers strictly less than 0.06032, bubble deformation due to a dominant hydrostatic pressure term does not occur at any moment during the bubble growth cycle. For bubble growth in which the Bond number is larger than 0.06032 , bubble deformation due to a dominant hydrostatic pressure term will occur.

\section{CONCLUSION}

A numerical treatment of the capillary equation is validated with measured profiles from quasistatic vapour bubble growth experiments. Due to a limited applicability of the hydrostatic pressure term, the solution procedure is deemed valid for bubble growth applications in which the Bond number with characteristic length equal to the bubble foot radius is less than or equal to 0.9941 .

This study demonstrates that a fixed base truncated spherical geometry rather than perfectly spherical more accurately describes bubble shape during bubble growth and that bubble shape evolution is strictly dependent on the Bond number. To summarize, bubbles of very different sizes with the same Bond number are shown to have the same shape profile.

The relationship between the Bond number and bubble shape may be explained by considering a relatively large Bond number implying a capillary length that is small relative to the cavity radius. This implies that the hydrostatic pressure has relative importance over the Laplace pressure drop at some depth from the bubble apex during its evolution. A hydrostatic pressure that dominates a Laplace pressure drop at a depth from the apex implies an inward pinching on the walls causing the bubble to deform from its truncated spherical minimum free energy shape. 
Furthermore, it is shown that for Bond numbers less than or equal to 0.06032 , the hydrostatic pressure does not dominate the Laplace pressure drop at any depth during the entire bubble growth cycle and consequently the bubble behaves as an approximate truncated sphere rising due to the elongation of a cylindrical neck. Future work will incorporate this into simplified analytical models for both diabatic and adiabatic bubble growths.

\section{ACKNOWLEDGMENTS}

The authors gratefully acknowledge the technical support of Rémi Pelletier of the Cégep de l'Outaouais and thank Francis Marois of the Université de Sherbrooke for his work in bubble image capturing during his 2012 summer internship.

${ }^{1}$ V. Dhir, "Nucleate and transition boiling heat transfer under pool and external flow conditions," Int. J. Heat Fluid Flow 12(4), 290-314 (1991).

${ }^{2}$ V. P. Carey, Liquid-Vapor Phase-Change Phenomena: An Introduction to the Thermophysics of Vaporization and Condensation Processes in Heat Transfer Equipment (Taylor and Francis, Hebron, KY, 1992).

${ }^{3} \mathrm{~V}$. Dhir, "Mechanistic prediction of nucleate boiling heat transfer-achievable or a hopeless task?," J. Heat Transfer 128, $1-12$ (2006).

${ }^{4}$ R. L. Judd and K. S. Hwang, "A comprehensive model for nucleate pool boiling heat transfer including microlayer evaporation," J. Heat Transfer 98, 623-629 (1976).

${ }^{5}$ W. Fritz, "Berechnung des maximalevolumens von dampfblasen," Phys. Z. 36, 379-384 (1935).

${ }^{6}$ N. Zuber, "Hydrodynamic aspects of boiling heat transfer," U S AEC Report AECU 4439, June 1959.

${ }^{7}$ R. Cole and H. L. Shulman, "Bubble departure diameters and subatmospheric pressures," AIChE Symp. Ser. 62, 6-16 (1966).

${ }^{8}$ R. Cole, "Bubble frequencies and bubble departures at subatmospheric pressures," AIChE J. 13, 779-783 (1967).

${ }^{9}$ M. K. Jensen and G. J. Memmel, "Evaluation of bubble diameter correlations," Proc. Int. Heat Transfer Conf. 4, 1907-1912 (1986).

${ }^{10}$ S. S. Kutateladze and I. I. Gogonin, "Growth rate and detachment diameter of a vapor bubble in free convection boiling of a saturated liquid," High Temp. 17, 667-671 (1979).

${ }^{11}$ S. Quan and J. Hua, "Numerical studies of bubble necking in viscous liquids," Phys. Rev. E 77, 066303 (2008).

${ }^{12}$ S. Di Bari and A. J. Robinson, "Experimental study of gas injected bubble growth from submerged orifices," Exp. Therm. Fluid Sci. 44, 124-137 (2013).

${ }^{13}$ G. Duhar and C. Colin, "Dynamics of bubble growth and detachment in a viscous shear flow," Phys. Fluids $\mathbf{1 8 ,}$ 077101-1-077101-13 (2006).

${ }^{14}$ A. J. Robinson, F. Lesage, and R. L. Judd, "Numerical method for spherical bubble growth in superheated liquids," Comput. Therm. Sci. 2(1), 19-31 (2010).

${ }^{15}$ A. K. Das, P. K. Das, and P. Saha, "Heat transfer during pool boiling based on evaporation from micro and macrolayer," Int. J. Heat Mass Transfer 49, 3487-3499 (2006).

${ }^{16}$ I. Chakraborty, B. Ray, G. Biswas, F. Durst, A. Sharma, and P. S. Ghoshdastidar, "Computational investigation on bubble detachment from submerged orifice in quiescent liquid under normal and reduced gravity," Phys. Fluids 21, 062103 (2009).

${ }^{17}$ Q. Bai and Y. Fujita, "Numerical simulation of bubble growth in nucleate boiling - Effects of system parameter," Multiphase Sci. Technol. 12(3-4), 195-214 (2000).

${ }^{18}$ H. Y. Yoon, S. Koshizuka, and Y. Oka, "Direct calculation of bubble growth, departure, and rise in nucleate pool boiling," Int. J. Multiphase Flow 27, 277-298 (2001).

${ }^{19} \mathrm{P}$. Genske and K. Stephan, "Numerical simulation of heat transfer during growth of single vapor bubbles in nucleate boiling," Int. J. Therm. Sci. 45(3), 299-309 (2006).

${ }^{20}$ T. Fuchs, J. Kern, and P. Stephan, "A transient nucleate boiling model including microscale effects and wall heat transfer," J. Heat Transfer 128(12), 1257-1265 (2006).

${ }^{21}$ J. Wu, V. K. Dhir, and J. Qian, "Numerical simulation of subcooled nucleate boiling by coupling level-set method with moving-mesh method," Numer. Heat Transfer, Part B 51(6), 535-563 (2007).

${ }^{22}$ A. Mukherjee and S. G. Kandlikar, "Numerical study of single bubbles with dynamic contact angle during nucleate pool boiling," Int. J. Heat Mass Transfer 50(1-2), 127-138 (2007).

${ }^{23}$ P. Stephan and T. Fuchs, "Local heat flow and temperature fluctuations in wall and fluid in nucleate boiling systems," Heat Mass Transfer 45, 919-928 (2009).

${ }^{24}$ B. K. Mori and D. W. Baines, "Bubble departure from cavities," Int. J. Heat Mass Transfer 44, 771-783 (2001).

${ }^{25}$ D. Gerlach, G. Biswas, F. Durst, and V. Kolobaric, "Quasi-static bubble formation on submerged orifices," Int. J. Heat Mass Transfer 48, 425-438 (2005).

${ }^{26}$ A. Faghri and Y. Zhang, Transport Phenomena in Multiphase Systems (Elsevier, Burlington, MA, 2006).

${ }^{27}$ C. W. Van der Geld, "The dynamics of a boiling bubble before and after detachment," Heat Mass Transfer 45, 831-846 (2009).

${ }^{28}$ V. V. Buwa, D. Gerlach, F. Durst, and E. Schülcker, "Numerical simulations of bubble formation on submerged orifices: Period-1 and period-2 bubbling regimes," Chem. Eng. Sci. 62, 7119-7132 (2007).

${ }^{29}$ J. C. Burton, R. Waldrep, and P. Taborek, "Scaling and instabilities in bubble pinch-off," Phys. Rev. Lett. 94, 184502 (2005). 
${ }^{30}$ N. C. Keim, P. Moller, W. W. Zhang, and S. R. Nagal, "Breakdown of air bubbles in water: Memory and breakdown of cylindrical symmetry," Phys. Rev. Lett. 97, 144503 (2006).

${ }^{31}$ S. T. Thoroddsen, T. G. Etoh, and K. Takehara, "Experiments on bubble pinch-off," Phys. Fluids 19, 042101-1-042101-29 (2007).

${ }^{32}$ S. T. Thoroddsen, T. G. Etoh, and K. Takehara, "High-speed imaging of drops and bubbles," Annu. Rev. Fluid Mech. 40, 257-285 (2008).

${ }^{33}$ E. Iacona, C. Herman, S. Chang, and Z. Liu, "Electric field effect on bubble detachment in reduced gravity environment," Exp. Therm. Fluid Sci. 31, 121-126 (2006).

${ }^{34}$ F. Chen, Y. Peng, Y. Z. Song, and M. Chen, "EHD behaviour of nitrogen bubble in DC electric fields," Exp. Therm. Fluid Sci. 32, 174-181 (2007)

${ }^{35}$ J. J. Nieuwland, M. L. Veenendaal, J. A. Kuipers, and W. P. M. Vanswaaij, "Bubble formation at a single orifice in gas-fluidised beds," Chem. Eng. Sci. 51(17), 4087-4102 (1996). 